\title{
Poynting Effects in Pantographic Metamaterial Captured via Multiscale DVC
}

\author{
Patrick Auger, ${ }^{1}$ Thomas Lavigne, ${ }^{1}$ Benjamin Smaniotto, ${ }^{1,2}$ \\ Mario Spagnuolo, ${ }^{3}$ Francesco dell'Isola ${ }^{3,4}$ and François Hild ${ }^{2}$
}

\begin{abstract}
Metamaterials are often studied for their peculiar mechanical properties. However, few 4D studies were conducted on 3D printed pantographs. This study aims at analyzing an in situ torsion test in a lab tomograph. The acquired scans were used to measure displacement fields via digital volume correlation. The final goal was to analyze the deformation mechanisms of an Inconel pantograph and to rationalize potential Poynting effects.
\end{abstract}

\section{Keywords}

Digital Volume Correlation (DVC); Metamaterials; Multiscale analyses; Torsion test; Uncertainty quantification. 


\section{Introduction}

The so-called Poynting effect is a finite-strain coupling observed in solid mechanics when dealing with shear deformations. This phenomenon is visible when, for instance, a cylinder is subjected to torsion and its axial length changes. The first experimental observations were reported by J.H. Poynting [1, $2,3]$. When axial elongation or shortening are observed, then the so-called Poynting effect is categorized as positive or negative, respectively. Classical (i.e., Cauchy) elasticity explains Poynting effects in homogeneous materials as due to geometrical nonlinearities [4]. Theoretical analyses based on strain gradient models show that, in some classes of heterogeneous and fibrous materials, the Poynting effect can pass from being positive to negative (or vice versa) when the material undergoes large deformations [5]. However, the understanding of the influence of material architecture on the Poynting effect is still at an embryonal stage. Filling this gap would allow for the design of heterogeneous artificial materials, known as metamaterials [6], with tailored Poynting effects.

The aim of this paper is to move one step toward a better understanding of Poynting effects in architectured materials. The attention is herein devoted to a special class of heterogeneous materials, namely so-called pantographic

\footnotetext{
${ }^{1}$ ENS Paris-Saclay, DGM-Department of Mechanical Engineering, Gif-sur-Yvette, France

${ }^{2}$ Université Paris-Saclay, ENS Paris-Saclay, CNRS, LMT-Laboratoire de Mécanique et Technologie, Gif-sur-Yvette, France

${ }^{3}$ International Research Center for the Mathematics and Mechanics of Complex Systems, Universitá degli Studi dell'Aquila, L'Aquila, Italy

${ }^{4}$ Dipartimento di Ingegneria Civile, Edile-Architettura e Ambientale, Universitá degli Studi dell'Aquila, L'Aquila, Italy

Corresponding author:

François Hild. Email: francois.hild@ens-paris-saclay.fr
} 
fabrics [7]. Pantographic fabrics are made up of two families of mutually orthogonal, equidistant and continuous fibers, arranged in two different planes and interconnected by cylindrical joints (henceforth called hinges), as shown in Figure 1. Their torsional behavior is experimentally characterized for the first time by means of multi-scale Digital Volume Correlation analyses applied to reconstructed volumes acquired during an in situ torsion test in an X-ray scanner, in addition to standard torque and axial force measurements.

Pantographic metamaterials were mostly studied from a modeling point of view (e.g., see Refs. $[8,9,10,11])$. In particular, the role of the interconnecting parts of the two fiber families was shown to be of primary importance when deriving the strain energy of such systems [12]. In the present work, special attention is devoted to their deformation in torsion. Experimental characterizations of such materials are less numerous and usually rely on global recordings such as the stroke and resultant force in bias extension tests [13]. Recently, it was shown that digital image correlation could be applied to monitor in quantitative ways the deformations of such metamaterials at different scales $[14,15]$. Out-of-plane motions occurring in shear tests were quantified via stereocorrelation $[16,17]$. Such technique may be applicable to torsion tests but would require a multiview setup [18] since rotation amplitudes as large as $180^{\circ}$ or more may occur [5]. Therefore, it was decided to perform an in situ torsion test.

The paper is organized as follows. First, the in situ torsion test is described, and all the various acquisition are presented. One post-processing step consists in measuring displacement fields for the 7 acquired tomographic scans via DVC. Second, given the large angular amplitudes experienced by the metamaterial, various additional steps had to be implemented for the FE-based DVC analyses to converge when direct registrations were sought. Further, the printed geometry was not close to its nominal model. An additional DVC step was then performed 
to backtrack the finite element model of the nominal geometry onto the printed configuration. All these operations enabled displacement fields to be measured at the macroscopic and mesoscopic scales of the metamaterial, and their uncertainties to be quantified. Third, from the measured displacement fields, the strain fields were computed and analyzed. In particular, two different Poynting effects are studied at the macro- and meso-scales.

\section{Torsion test}

The sample used herein was produced by additive manufacturing via Selective Laser Melting (SLM). For a complete description of the method used for producing the sample, the reader is referred to Ref. [19]. The employed material was Inconel 625 (i.e., nickel-chromium superalloy). The dimensions of the sample were chosen such that one side was three times longer than the other one. Specifically, the pantographic structure (see Figure 1(a-b)) had the shape of a rectangle of sides $3 \mathrm{~cm}$ and $9 \mathrm{~cm}$. The 'fibers' had a square section side $1 \mathrm{~mm}$ long, and the connecting hinges were cylinders whose diameter was $0.2 \mathrm{~mm}$, and height $1 \mathrm{~mm}$ (see Figure 1(c)). These values are nominal and were used to design the pantograph in the $\mathrm{CAD}$ modeler. The resulting CAD file was then used to print the sample. 


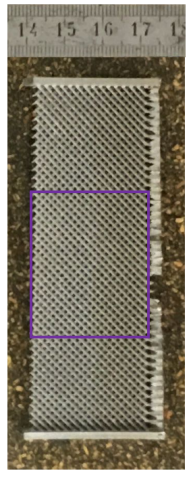

(a)

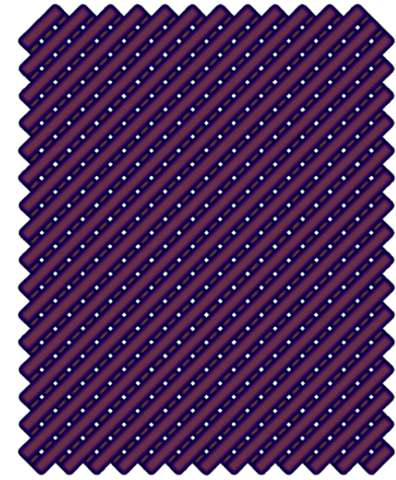

(b)

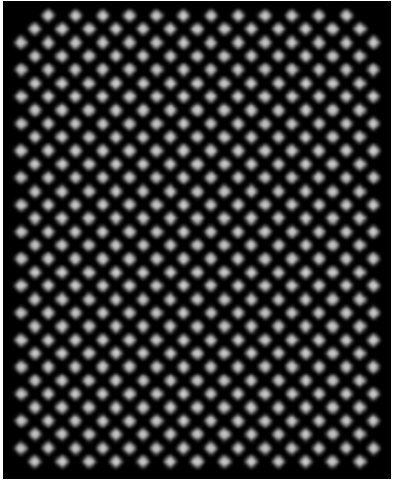

(c)

Figure 1. (a) Printed pantograph (the ruler is in $\mathrm{mm}$ ). The purple box corresponds to the analyzed ROI via DVC. (b) Nominal geometry of the ROI. (c) Corresponding mid-height section showing the hinges connecting the two families of orthogonal beams

The torsion test was monitored using micro-computed tomography. Such imaging technique is non-intrusive and provides 3D images of the sample tested in situ [20]. The torsion torque was applied by two angular actuators of the in situ testing machine, both controlled in angular position. The levels of torque and axial force were regularly recorded. The test was conducted until reaching an angular amplitude of $\theta=180^{\circ}$ between the bottom and the top platens (Figure 2). A last step consisted in getting back to the initial angular position. 


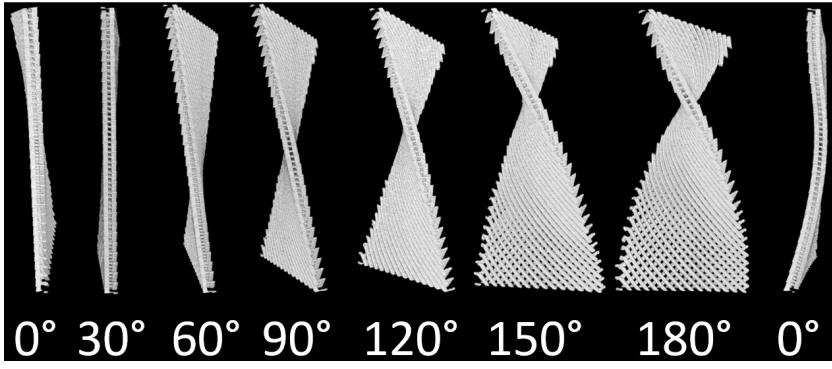

(a)

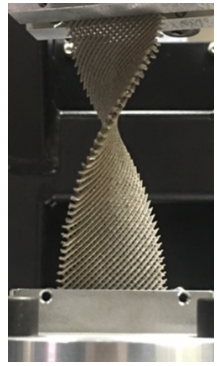

(b)

Figure 2. (a) 3D renderings of the tested sample for different angular amplitudes $\theta$ in torsion. (b) In situ view for the $180^{\circ}$ amplitude

The series of 600 radiographs per scan (i.e., sinograms) were used to reconstruct $3 \mathrm{D}$ images of the sample during the test (Figure 2). The hardware parameters of the in situ setup are gathered in Table 1. Once cropped, the reconstructed volumes covered $43.5 \times 42 \times 48.3 \mathrm{~mm}^{3}$ with a $55 \mu \mathrm{m}$ resolution . 
Table 1. DVC hardware parameter

\begin{tabular}{|c|c|}
\hline Tomograph & North Star Imaging X50+ \\
\hline $\mathrm{X}$-ray source & XRayWorX XWT-240-CT \\
\hline Target / Anode & $\mathrm{W}$ (reflection mode) \\
\hline Filter & $\mathrm{Cu}(0.5 \mathrm{~mm})$ \\
\hline Voltage & $200 \mathrm{kV}$ \\
\hline Current & $100 \mu \mathrm{A}$ \\
\hline Focal spot size & $5 \mu \mathrm{m}$ \\
\hline Tube to detector & $510 \mathrm{~mm}$ \\
\hline Tube to object & $185 \mathrm{~mm}$ \\
\hline Detector & Dexela 2923 \\
\hline Definition & $1536 \times 1944$ pixels $(2 \times 2$ binning $)$ \\
\hline Number of projections & 600 \\
\hline Angular amplitude & $360^{\circ}$ \\
\hline Frame average & 8 per projection \\
\hline Frame rate & $6 \mathrm{fps}$ \\
\hline Acquisition duration & $21 \min 25 \mathrm{~s}$ \\
\hline Reconstruction algorithm & filtered back-projection \\
\hline Gray Levels amplitude & 8 bits \\
\hline Volume size & $791 \times 764 \times 879$ voxels $($ after crop $)$ \\
\hline Field of view & $43.5 \times 42 \times 48.3 \mathrm{~mm}^{3}$ (after crop) \\
\hline Image scale & $55 \mu \mathrm{m} /$ voxel \\
\hline Pattern & see Figure 4 \\
\hline
\end{tabular}

Figure 3 shows the change of torque and axial forces during the loading and unloading parts of the test. The small relaxations in the torque and axial force correspond to scan acquisitions. An axial force was observed (Figure 3(b)), which 
is due to the Poynting effect [5]. In the present case, the Poynting effect should consist of an elongation or shortening of the sample as a result of torsion. However, having clamped the short edges of the pantograph to maintain a constant length during the test, such elongation/shortening was prohibited and a reaction force was measured. From Figure 3, three different regimes are observed in the loading part. First, a quasi-linear part $\left(\theta \in\left[0^{\circ}, 30^{\circ}\right]\right)$. Then, the torque increases non-linearly while the force still increases quasi-linearly but with a lower slope $\left(\theta \in\left[30^{\circ}, 70^{\circ}\right]\right)$ due to the plastic behavior of the alloy (Figure 2$)$. The force reaches a maximum level at $\theta \approx 70^{\circ}$, and slowly decreases quasi-linearly while the torque tends to saturate $\left(\theta \in\left[70^{\circ}, 180^{\circ}\right]\right)$. These three different regimes illustrate the existence of both direct and reverse Poynting effects during the torsion test.

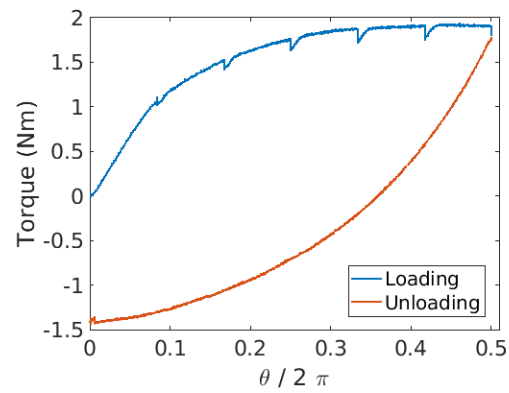

(a)

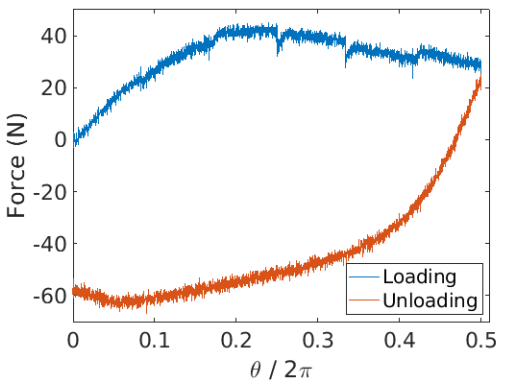

(b)

Figure 3. Torsion torque (a) and resultant force (b) during the loading and unloading parts of the test

In the loading part shown in Figure 3(b), the axial force initially increases (i.e., Poynting effect) and then begins to decrease. In the present test, such force never becomes negative, which would correspond to reverse Poynting effect. However, it is likely that some combinations of rigidities of the beams and hinges would induce reverse Poynting effect. 
The last part of the test was a full angular reversal (Figure 3). The first step corresponds to linear unloading, with a slope similar to the beginning of the loading curve (this is especially true for the torque, see Figure 3(a)). Then there is reverse plasticity that induced residual deformations of the sample. Having imaged 7 deformed configurations (Figure 2(a)), displacement fields were measured via DVC. Various steps had to be followed to capture the kinematic details of the torsion test.

\section{Different DVC Steps}

As the initial sample was already deformed by the printing method (Figure 4(a)), a preliminary DVC analysis was conducted between the image of the nominal geometry and that of the printed shape to backtrack the mesh that was constructed on the nominal geometry. DVC was also run incrementally on the different deformed configurations (Figure 2), and then directly, both with mechanical regularization [21]. The latter was required since virtually no contrast existed within the solid phase (Figure 4). In the present case, so-called Hencky-type elasticity was assumed (i.e., only the displacement increments from one configuration to the next were penalized [22]). 


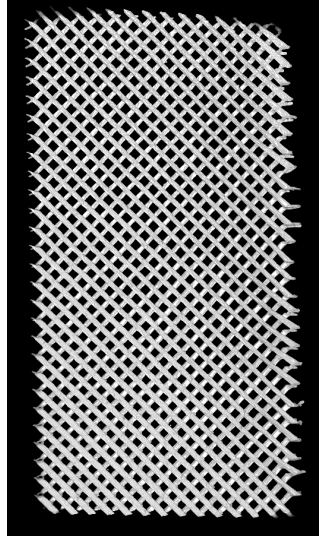

(a)

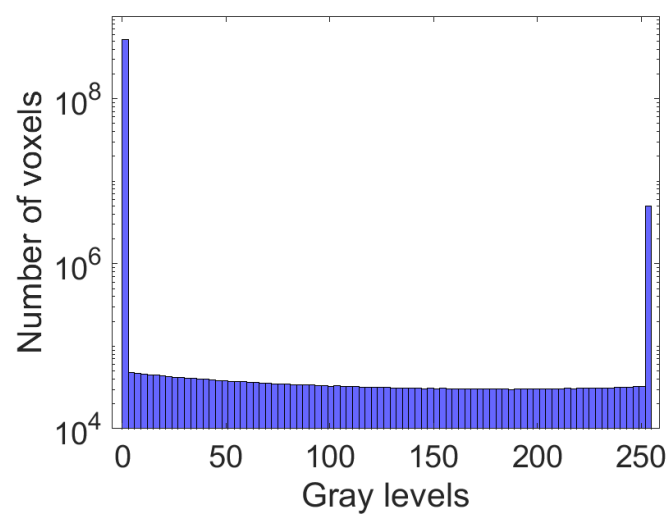

(b)

Figure 4. (a) $3 D$ rendering of the volume in the reference configuration.

(b) Corresponding gray level histogram

The volumes were processed using the Correli 3.0 framework [23] in which the elastic regularization was implemented (Table 2) in addition to all the algorithmic enhancements discussed in the sequel.

Table 2. DVC analysis parameters

\begin{tabular}{|l|l|}
\hline \hline DIC software & Correli 3.0 [23] \\
Image filtering & none \\
Element length (mean) & 27,24 and 8 voxels \\
Shape functions & linear (T4) \\
Meshes & see Figures 7 and 11 \\
Matching criterion & penalized sum of squared differences [21] \\
Regularization length & variable (see text) \\
Interpolant & cubic \\
Displacement noise floor & see Figure 12 \\
Strain noise floor & see Figure 13 \\
\hline
\end{tabular}




\section{Initialization and Large Rotations}

The temporal sampling of the various scans and the fact that large rotations occurred required some special care to be exercised when running DVC analyses. Given the angular amplitudes between two consecutive scans (Figure 2), DVC analyses were first run incrementally, namely, the deformed configuration of a given analysis became the reference configuration of the next one (and so on). The mesh was deformed according to the incremental nodal displacements so that Lagrangian estimates were obtained. Further, each DVC analysis was initialized since the overall kinematics of a torsion test could be guessed. In the present case, for each section containing nodes of the FE mesh (Figure 7), a 2D analysis was run between the reference and deformed configurations (Figure 5(a)). Three degrees of freedom were sought, namely, two (in-plane) translations and one (out of plane) rotation via integrated DIC [24]. Once they were obtained, the displacements were computed for each node of the considered section. As expected, the rotation amplitude varied linearly with the height (Figure 5(b))
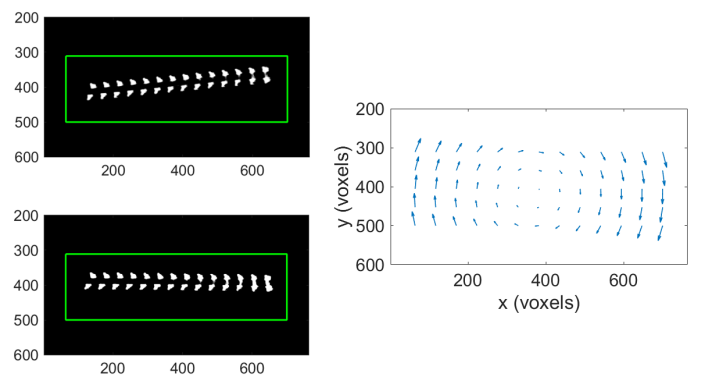

(a)

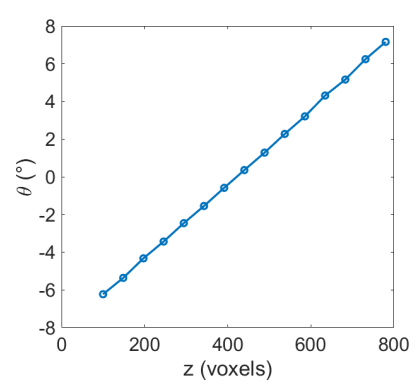

(b)

Figure 5. (a) Reference (top) and deformed (bottom) configurations for a 2D DIC analysis and estimated nodal displacements. (b) Change of the rotation angle with the sample height for a rotation amplitude of $30^{\circ}$ 
Once the calculation were initialized with the previous fields, the convergence speed was improved very significantly (i.e., in the present case a factor 4 reduction was observed). No additional steps were required to run incremental analyses. The drawback of the latter ones is that the measurement uncertainties are cumulated. To avoid such phenomenon, direct calculations may be run when initialized with incremental (and cumulated) calculations. However, when the rotation amplitudes increase, one additional step has to be performed [25, 26, 27] since the usual modification of the Gauss-Newton scheme (i.e., the Hessian matrix written with the gradient of the volume in the deformed configuration corrected by the measured displacement is approximated by that in the reference configuration) no longer applies.

In the following analyses, the route proposed by Guery et al. [25] was selected. It consists in evaluating the deformation gradient tensor $\mathbf{F}$ for nodes since the nodal displacement corrections are to be multiplied by $\mathbf{F}$. The tensor $\mathbf{F}$ is generally evaluated at integration points. In the present case, it was extrapolated at nodes via weighted averages. With such implementation, all direct calculations reported herein converged in less than 100 iterations. The best iteration (i.e., leading to the lowest RMS gray level residual) was kept once the L2-norm of the displacement corrections was less than $10^{-3}$ voxel.

\section{Feasibility Analysis}

The very first step consisted in performing a feasibility analysis for DVC runs. A mesh encompassing the reference configuration (Figure 6(top)) was constructed (Figure 7). Because of printing defects, the reference configuration was deformed with respect to the nominal geometry (Figure 6). Consequently, many elements did not contain any part of the pantograph. Because the DVC calculations were mechanically regularized [21], such situation was tractable and convergence was 
achieved in less than 30 iterations per step. However, the displacement field was only mechanically controlled in those zones.
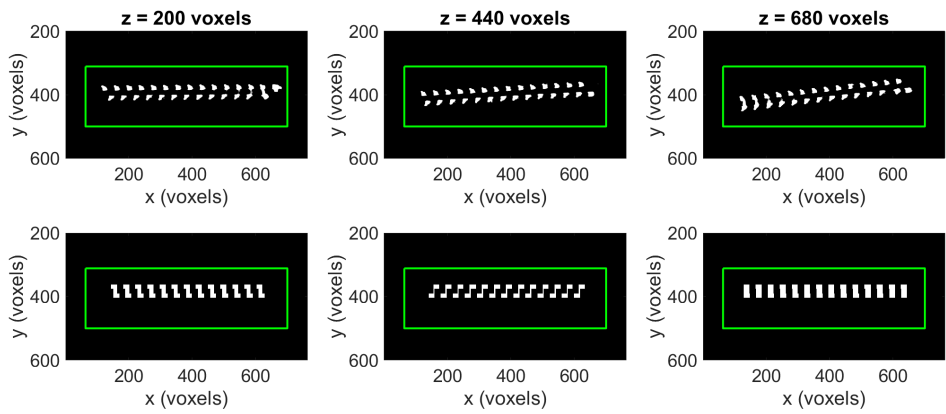

Figure 6. Sections of the reference (top) and nominal (bottom) configurations. The green boxes depict the external boundaries of the first (auxiliary) mesh (Figure 7)

This first (auxiliary) mesh was structured and made of 4-noded elements with linear shape functions (Figure 7). Its mean size, which was measured as the cubic root of the mean element volume, was equal to 27 voxels. The regularization length $\ell_{r}$ was equal to 150 voxels, which was close to the thickness of the meshed volume (i.e., 200 voxels). This choice was made to allow the DVC code to converge even though many elements were not containing any part of the studied pantograph.

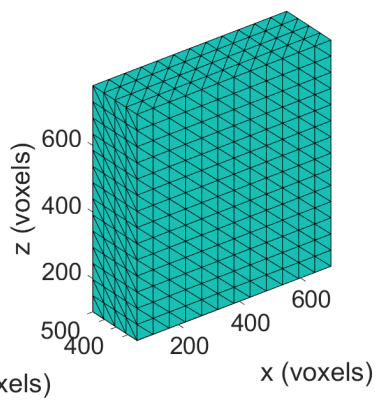

Figure 7. First (auxiliary) mesh constructed to encompass the printed configuration (Figure 6(top)) 
Figure 8 shows the displacement fields for the six analyzed scans along the direction orthogonal to the pantograph plane. The results are shown on the deformed configurations. It is worth noting that the displacement amplitudes are very large and explain why special care (i.e., initialization and regularization) had to be exercised to obtain such results.

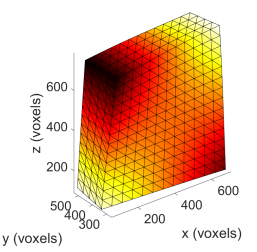

(a) $\theta=30^{\circ}$

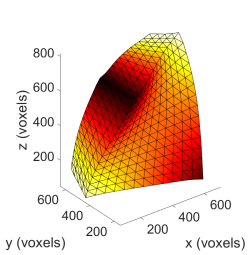

(d) $\theta=120^{\circ}$
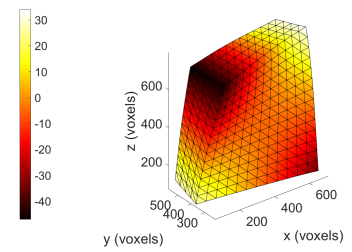

(b) $\theta=60^{\circ}$
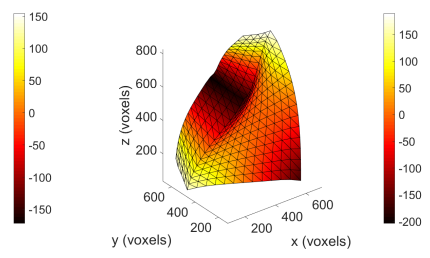

(e) $\theta=150^{\circ}$

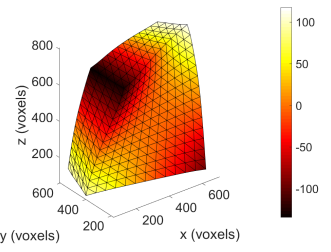

(c) $\theta=90^{\circ}$

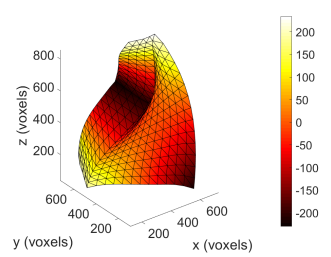

(f) $\theta=180^{\circ}$

Figure 8. Displacement fields in the $y$ direction (expressed in voxels) for different angular amplitudes. The fields are displayed on the deformed configurations

Having proven that DVC analysis could be run under such challenging conditions, the next step was to adapt the mesh to the underlying microstructure. It was performed thanks to a backtracking procedure that also uses DVC analyses [22, 28].

\section{Backtracking Meshes}

The first step of the backtracking procedure was to have access to the nominal volume, namely, the $3 \mathrm{D}$ image of the nominal configuration. In the present case, it was constructed by first creating a simplified mesh of the pantograph 
(Figure 9). This mesh was made of assemblies of elementary cells (e.g., hinges, hinges + beams, end cells). Since independent cells were assembled, one key operation was the deletion of double nodes within the connectivity matrix so that the displacements be continuous over the whole mesh. Had such operation failed, DVC runs would not have converged since the elements were too small (i.e., 8 voxels on average) with the data at hand.
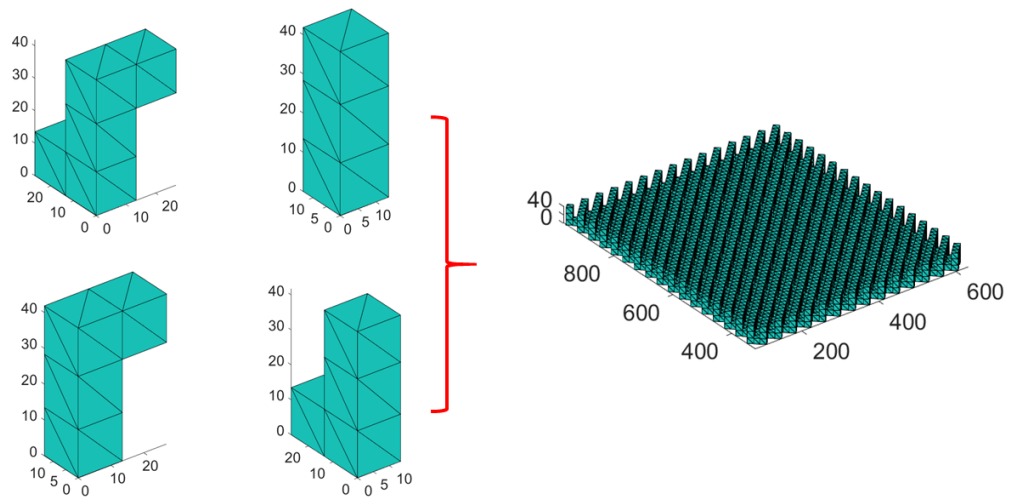

Figure 9. Schematic view of the construction of the nominal mesh at the mesoscale from the assembly of elementary cells

Once this mesh was available, the next operation was to backtrack it to fit as best as possible the printed configuration. Figure 10 summarizes the different steps. This backtracking operation required the knowledge of the 3D volume of the nominal configuration. All voxels belonging to the medium were assigned a gray level of 200, and the other ones kept a zero level. A Gaussian filter of radius 2 voxels was then applied to have a less sharp image that was easier to register than the one with sharp edges. Once the two volumes were available, it was possible to run a DVC analysis between real world data (i.e., the printed configuration) and virtual data (i.e., the nominal configuration). This registration required an auxiliary mesh, which corresponded in the present case to the mesh used in the feasibility analysis (Figure 7). A large regularization 
length was considered to enable for convergence (i.e., $\ell_{r}=150$ voxels) for the same reasons as before.

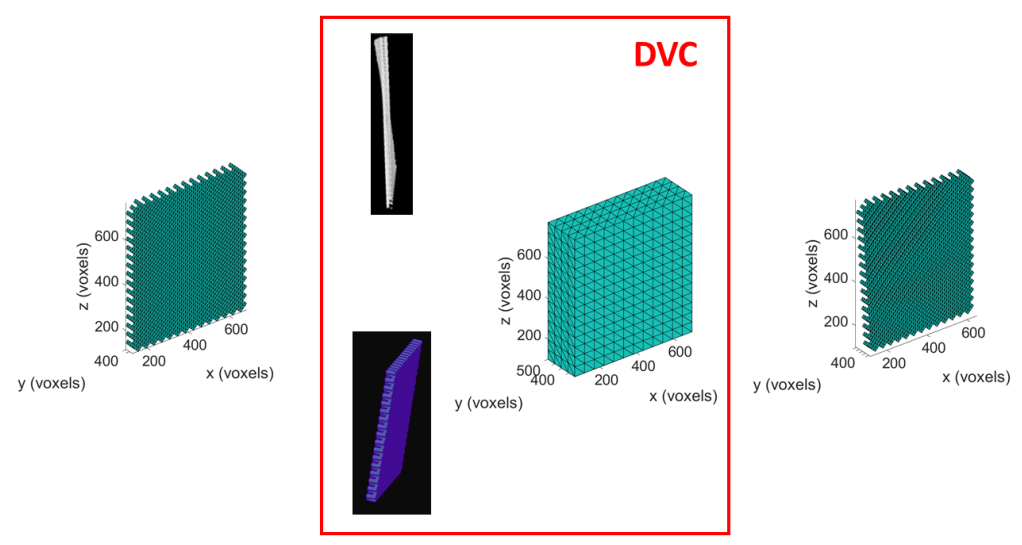

Figure 10. Schematic view of the backtracking procedure enabling nominal meshes (left) to be adapted to the printed configuration (right) via DVC analyses

Once convergence was achieved, the displacement field of the nominal configuration was known with respect to the printed configuration. It then sufficed to deform the auxiliary mesh according to the measured displacement field, to interpolate the displacement field on the nodes of the nominal mesh at the mesoscopic scale, and backtrack it by deforming it back to the printed configuration. This operation was also applied to a new mesh at the macroscopic scale that now encompassed better that at the mesoscopic scale (Figure 11). The mean element length of the new macroscopic mesh was equal to 24 voxels. 


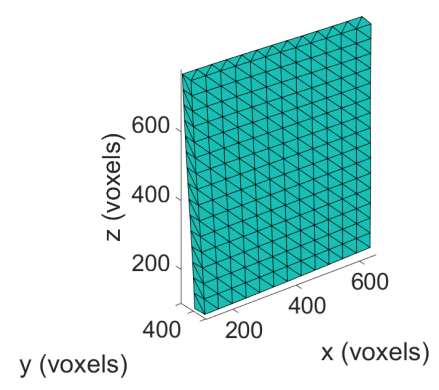

(a)

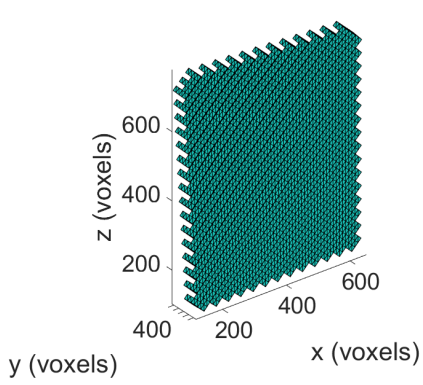

(b)

Figure 11. Backtracked macroscale (a) and mesoscale (b) meshes to the printed configuration

\section{Uncertainty Quantification}

The following uncertainty quantification was based on two scans that were acquired when the sample was in the reference configuration. A small rigid body rotation was applied by the two rotational actuators of the in situ testing machine. DVC analyses were run with the three meshes (Figures 7 and 11) for different regularization lengths $\ell_{r}$ ranging from 30 to 300 voxels. The rigid body motions were subtracted from the measured displacement fields, the standard

deviations of each nodal displacement component was estimated and its mean $\sigma_{u}$ is reported in Figure 12. 


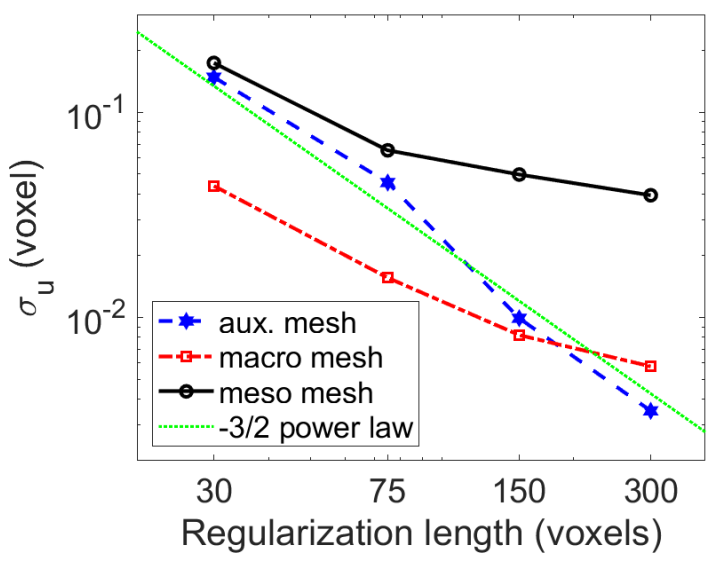

Figure 12. Standard displacement uncertainties $\sigma_{u}$ vs. regularization lengths $\ell_{r}$ for the three meshes (Figures 7 and 11). The dotted line depicts the power $-3 / 2$ trend

For each discretization, the larger the regularization, the lower the standard displacement uncertainty $\sigma_{u}$. This general trend was expected as larger regularization lengths correspond to more weight put on the mechanical regularized that is able to filter out more noise. It is worth noting that the $-3 / 2$ power dependence of $\sigma_{u}$ is not observed for the two backtracked meshes, which indicates that other sources of uncertainties are more prevalent. Such observations were already reported for other experimental configurations [29]. Conversely, the $-3 / 2$ power law captures the overall trends of the auxiliary mesh (Figure 7). In that case, a majority of voxels lied in the background, which was more influenced by acquisition noise and less by other artifacts.

The auxiliary mesh (Figure 7) yield measurement uncertainties close to those of the macroscale mesh for large regularization lengths for which most of the fluctuations are controlled by mechanical regularization. For smaller regularization lengths, the measurement uncertainties have the same order of magnitude as the mesh at the mesoscale since mechanical regularization is penalizing less the DVC results. It is worth remembering that this mesh cannot 
be used with no mechanical regularization since zones with no contrast prevent the convergence of the DVC code.

For the macroscale mesh (Figure 11(a)), the measurement uncertainties are very low in comparison with other microstructures [30]. This difference is due to the fact that in that case there are numerous instances of very high contrast that induces very small uncertainties of DVC analyses. Conversely, for the mesoscopic mesh (Figure 11(b)), the standard displacement uncertainties are 4 times higher in comparison to the macroscopic mesh. This increase is due to the fact that there is virtually no contrast within the solid phase, which made the DVC analyses at the mesoscale very challenging and called for the use of mechanical regularization. These results show that the regularization should not be too small if fine discretizations were to be used.

In the last part of the paper, two strain components will be reported, namely, the maximum principal strain $\epsilon_{1}$ and the shear strain $\epsilon_{x y}$. Their corresponding uncertainties are shown in Figure 13. For any discretization, very low levels are observed for the largest regularization length. Such trend shows the benefit of mechanical regularization that acts as a low pass filter for kinematic measurements. There is an even more pronounced difference (i.e., at least one order of magnitude) between both meshes at the macroscale and the one at the mesoscale, which again proves that the analyses at the mesoscale were very challenging. 


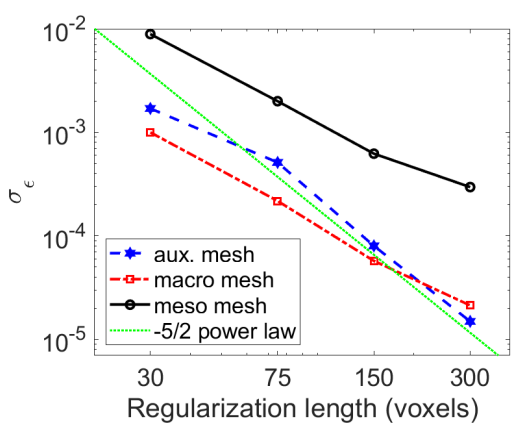

(a)

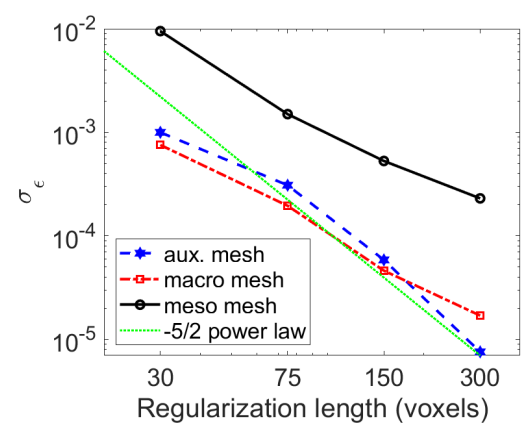

(b)

Figure 13. Standard strain uncertainties $\sigma_{\epsilon}$ (for the maximum principal strain $\epsilon_{1}(\mathrm{a}$ ) and shear strain $\left.\epsilon_{x y}(\mathrm{~b})\right)$ vs. regularization lengths $\ell_{r}$ for the three meshes (Figures 7 and 11). The dotted line depicts the power $-5 / 2$ trend

Since the power $-3 / 2$ dependence of the displacement uncertainty was recovered for the auxiliary mesh, it was expected that the strain uncertainty would follow a power $-5 / 2$ law of the regularization length. Such dependence is observed for both meshes at the macroscopic scale. As anticipated from the displacement uncertainties of the mesoscale discretization, the previous trend is not observed for the corresponding strain uncertainty.

\section{Macroscopic Analyses}

Using the backtracked macroscopic mesh (Figure 11(a)), direct DVC analyses could be run. They started with a large regularization length (i.e., $\ell_{r}=$ 300 voxels) and were initialized with the results of the displacements from the feasibility analysis interpolated over the new mesh. Once convergence was obtained, the regularization length could be gradually decreased in a relaxation procedure [21].

Figure 14 shows the change of the gray level residuals with the angular amplitude in the torsion test. The higher the amplitude, the larger the residuals. 
This trend indicates that as the angular amplitude increases, the selected kinematics associated with the macroscopic mesh is no longer able to capture the actual motions. Such result is not a shortcoming of the DVC procedure itself, but rather it is related to the kinematic hypotheses made in the analyses. It is also interesting to note that as the regularization length was decreased, so did the RMS residuals (i.e., less constraints were prescribed to the minimization of the global cost function). Conversely, the residuals with the largest regularization length were very high, which disqualified such results since too much weight was put on a regularization hypothesis that was no longer valid (i.e., plasticity had occurred in a large part of the pantograph).

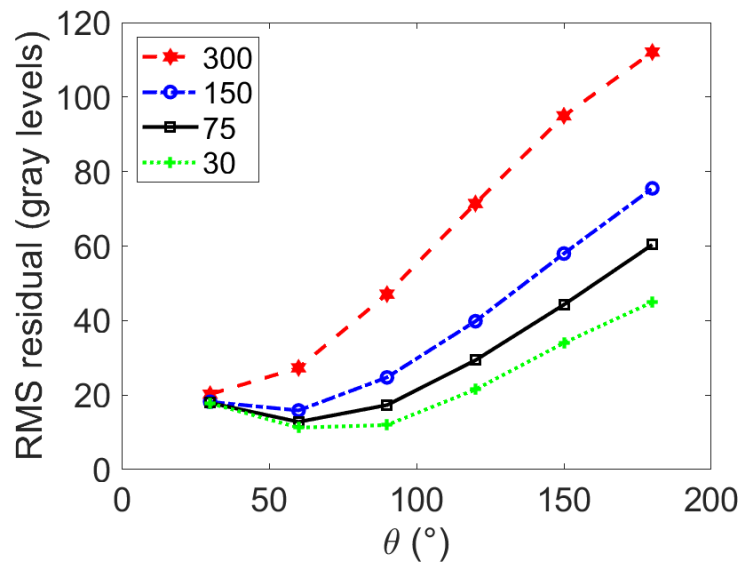

Figure 14. RMS gray level residuals vs. angular amplitude for different regularization lengths in DVC analyses using the macroscopic mesh

In the present case, DVC analyses could be run with no regularization at all (i.e., $\ell_{r}=0$ ). The RMS residuals had levels virtually identical to those observed when $\ell_{r}=30$ voxels. Even though the smallest regularization lengths led to the lowest residuals, the following results are reported when $\ell_{r}=75$ voxels for comparison purposes with the analyses at the mesoscale that could not be conducted with too small regularization lengths (Figure 12). In Figure 15, the 
displacement fields are reported for the six analyzed scans along the direction orthogonal to the pantograph plane. These new results were obtained via direct DVC runs and converged thanks to the initialization strategy and the use of the modified displacement increments with the nodal deformation gradient tensor.

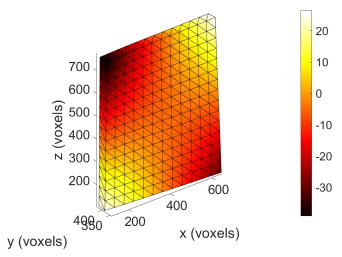

(a) $\theta=30^{\circ}$

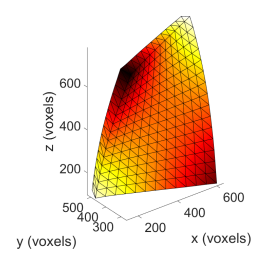

(d) $\theta=120^{\circ}$

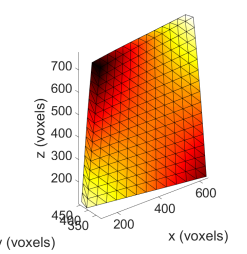

(b) $\theta=60^{\circ}$
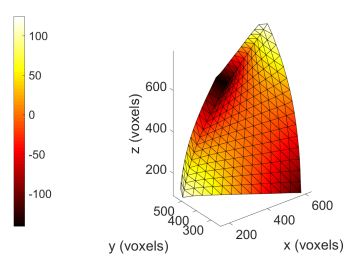

(e) $\theta=150^{\circ}$
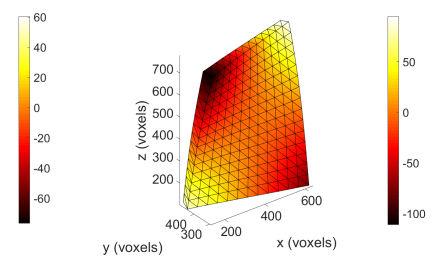

(c) $\theta=90^{\circ}$
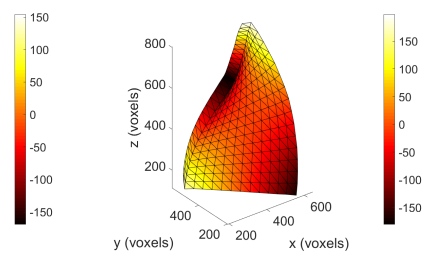

(f) $\theta=180^{\circ}$

Figure 15. Displacement fields in the $y$ direction (expressed in voxels) for different angular amplitudes for DVC analyses at the macroscale ( $\ell_{r}=75$ voxels). The fields are displayed on the deformed configurations

\section{Mesoscopic Analyses}

Having access to displacement fields at the macroscale, the following analyses were conducted with the mesoscale mesh (Figure 11(b)). The same relaxation strategy was applied to the present case. The corresponding changes of the RMS gray level residuals are shown in Figure 16. The lowest regularization length (i.e., $\ell_{r}=75$ voxels) led to residuals that remained at the same levels for most of the test, and only degraded for the largest rotation amplitude. 


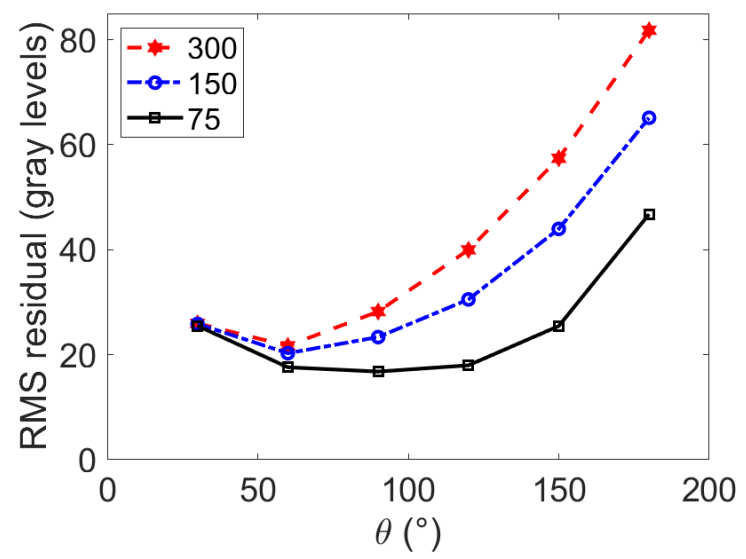

Figure 16. RMS gray level residuals vs. angular amplitude for different regularization lengths in DVC analyses using the mesoscopic mesh

Directly comparing the levels of the RMS residuals reported for the macroscale (Figure 14) and mesoscale (Figure 16) meshes was not possible since the meshed regions of interest were not identical. It was however possible to interpolate the measured displacement fields at the macroscale with the mesoscale mesh since the nodes of the latter lied inside the region of interest of the macroscale mesh. The RMS residuals were evaluated with this new displacement field for the region of interest of the mesoscopic mesh. Figure 17 shows that the results at the mesoscale generally led to lower gray level residuals, which means that they were more trustworthy. Conversely, for the first two rotation amplitudes, the difference between mesoscale and macroscale results was not very important. This observation shows that in the first two regimes of the loading curves (Figure 3) both descriptions faithfully capture the experimental kinematics. In the third regime, this is no longer the case, and the lowest regularization length leads to the best results. Such differences are due to the hinges, as will be shown in the sequel. 


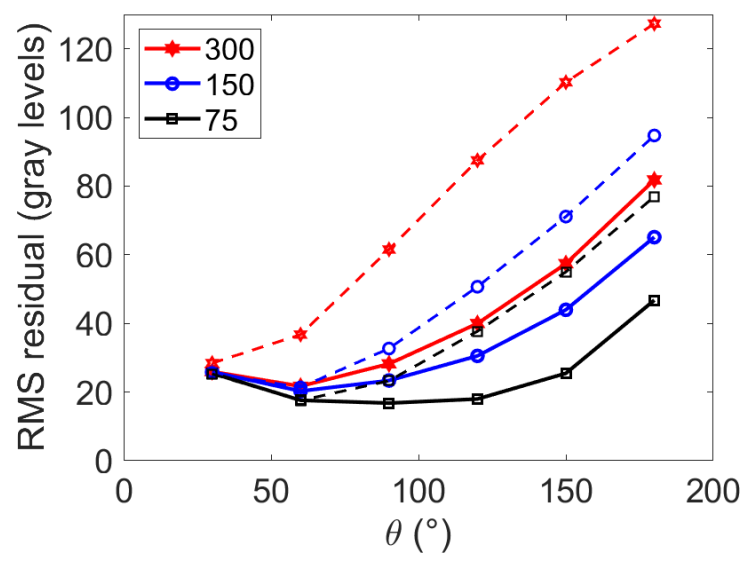

Figure 17. RMS gray level residuals vs. angular amplitude for different regularization lengths in DVC analyses using the macroscopic (dashed lines) and mesoscopic (solid lines) meshes

Figure 18 shows the displacement fields for the six analyzed scans along the direction orthogonal to the pantograph plane. They will be further processed to evaluate various strain fields in the following section. 


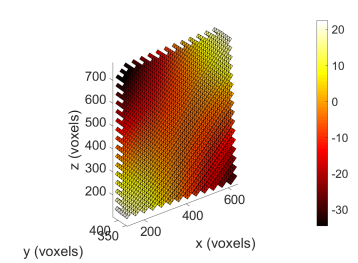

(a) $\theta=30^{\circ}$

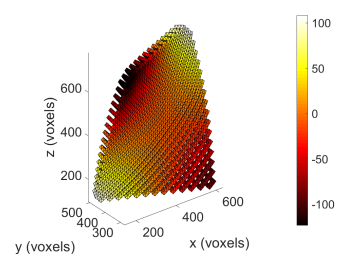

(d) $\theta=120^{\circ}$

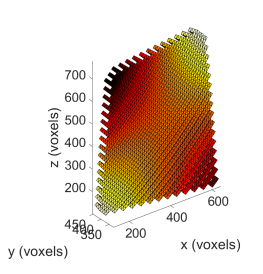

(b) $\theta=60^{\circ}$

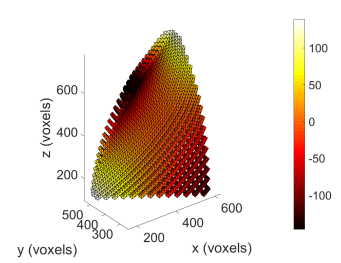

(e) $\theta=150^{\circ}$

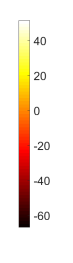

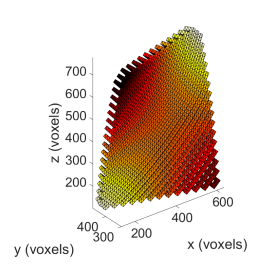

(c) $\theta=90^{\circ}$

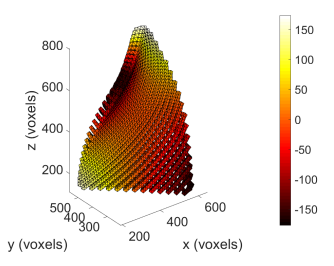

(f) $\theta=180^{\circ}$

Figure 18. Displacement fields in the $y$ direction (expressed in voxels) for different angular amplitudes for DVC analyses at the mesoscale $\left(\ell_{r}=75\right.$ voxels). The fields are displayed on the deformed configurations

\section{Analysis of the results}

\section{Strain Fields}

The results obtained with the mesh at the mesoscale and the smallest regularization length $\ell_{r}=75$ voxels are further discussed in terms of strain fields. In the present case, the strains were determined by exact differentiation of the shape functions of the T4 elements (i.e., the deformation gradient tensor

$\mathbf{F}$ is uniform in each element since the displacement interpolation is linear). No filtering was applied. Since large transformations occurred, the Green Lagrange measure was selected.

Figure 19 shows the maximum principal strain field for the six analyzed scans. In the beams, the strains remained small, especially when getting close to the center of the specimen. Conversely, higher levels are observed when moving away 
from the center. Even higher levels occurred in the hinges, which concentrated most of the deformation especially at the end of the test (i.e., $\theta>90^{\circ}$ ).

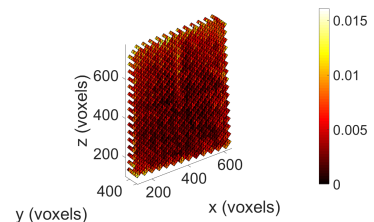

(a) $\theta=30^{\circ}$

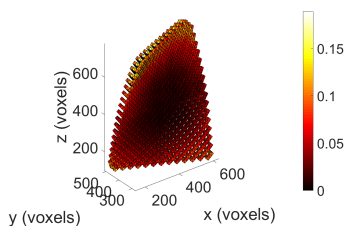

(d) $\theta=120^{\circ}$

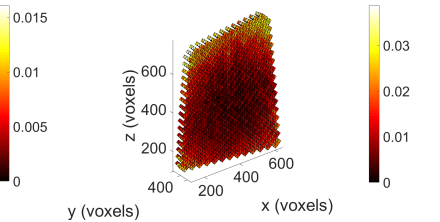

(b) $\theta=60^{\circ}$

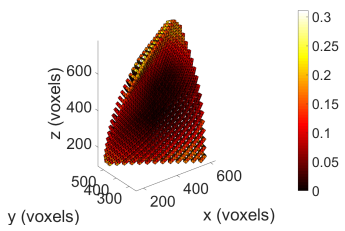

(e) $\theta=150^{\circ}$

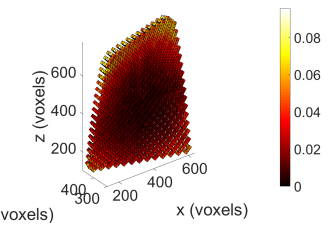

(c) $\theta=90^{\circ}$

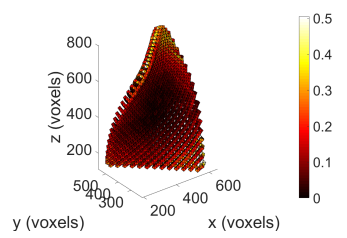

(f) $\theta=180^{\circ}$

Figure 19. Maximum principal strain $\epsilon_{1}$ fields for the different angular amplitudes for DVC analyses at the mesoscale ( $\ell_{r}=75$ voxels). The fields are displayed on the deformed configurations

To further analyze this last observation, the shear strain $\epsilon_{x y}$ fields are reported in Figure 20. The viewpoint was changed to focus more on the hinges farther away from the specimen center. For the first rotation amplitude, the shear strains in the hinges were very close to the yield strain of the material (i.e., $\approx 1 \%$ ). From the second angular amplitude on, the strains significantly concentrated in the hinges, and reach rather high absolute levels (i.e., up to 0.4 for the last scan). Consequently, the overall behavior of the pantographic structure is very dependent on the response of the hinges to the applied load. 


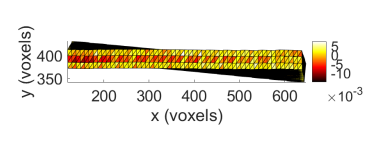

(a) $\theta=30^{\circ}$

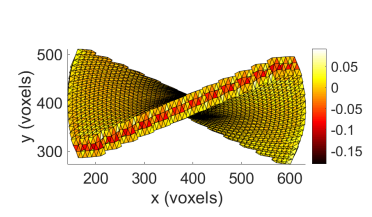

(d) $\theta=120^{\circ}$

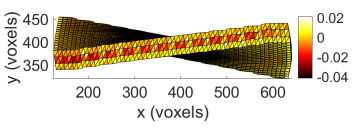

(b) $\theta=60^{\circ}$

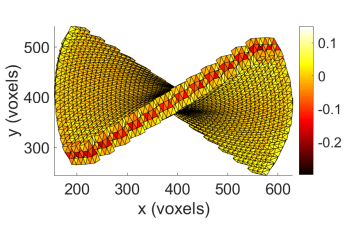

(e) $\theta=150^{\circ}$

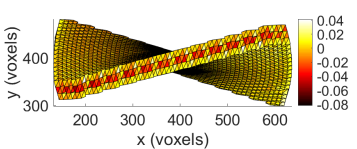

(c) $\theta=90^{\circ}$

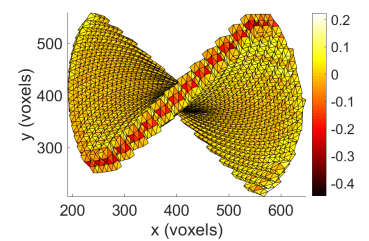

(f) $\theta=180^{\circ}$

Figure 20. Strain $\epsilon_{x y}$ fields for the different angular amplitudes for DVC analyses at the mesoscale ( $\ell_{r}=75$ voxels). The fields are displayed on the deformed configurations

\section{Meso-Poynting Effect}

In this subsection, some results are shown for the elements encompassing the hinges. This type of analysis, at the mesolevel, was allowed by the DVC analyses reported herein, and makes possible the direct observation of some mechanisms that otherwise can only be inferred from a theoretical point of view. In particular, because the hinges were subjected to multiaxial loadings, they may have experienced torsion and possibly Poynting effects at this reduced scale, which is referred to as meso-Poynting effect.

The following results are only displayed on the elements containing the hinges. For each hinge, the reported strains were averaged over the six elements that described their kinematics. Figure 21 shows the shear strain $\epsilon_{x y}$ levels for the six angular positions. For the last four angular positions, the strain distributions were identical, but their overall magnitude increased linearly with the applied 
angle. Macroscopically, it corresponds to the third regime in which the torque flattens, and the axial force decreases linearly with the applied angle. The shear strains were rather low in the first two angular positions, and close to the yield strain of the bulk material (i.e., $\approx 1 \%$ ) for the second angle.

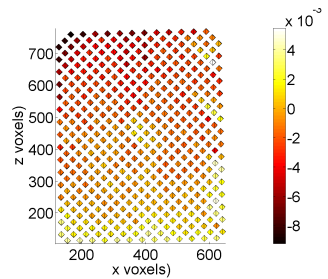

(a) $\theta=30^{\circ}$

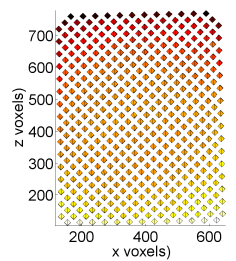

(d) $\theta=120^{\circ}$

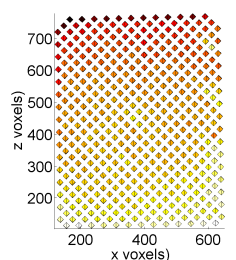

(b) $\theta=60^{\circ}$

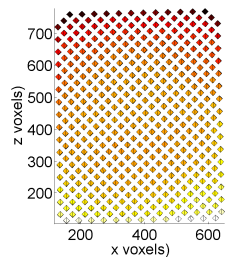

(e) $\theta=150^{\circ}$

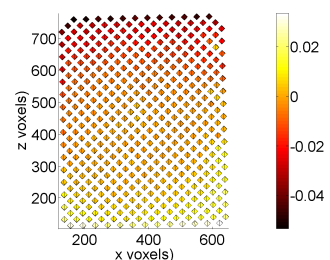

(c) $\theta=90^{\circ}$

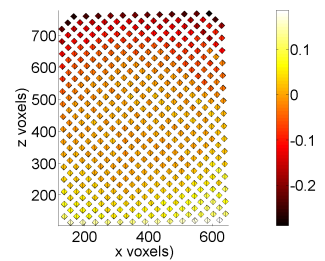

(f) $\theta=180^{\circ}$

Figure 21. Average strains $\epsilon_{x y}$ in the hinges for the different angular amplitudes. The fields are displayed on the initial configuration

The shear strain $\epsilon_{x y}$ represents shear of hinges considered as cylinders with their symmetry axis along $y$. This information can be interpreted by considering the results reported in Ref. [31]. The influence of the shear strain of the hinges was studied to characterize the mechanical behavior of the pantographic structures. This shear strain induced relative displacements between the two 'fibers' that are connected by the hinge itself.

Another field of interest is the equivalent von Mises strain $\epsilon_{e q}$, which was evaluated from the deviator of the total strain tensor. This field is reported for the six angular positions in Figure 22. Compared to Figure 21, the $\epsilon_{e q}$ fields are more concentrated on the upper and lower parts, and their levels are significantly 
higher. These last fields show that plasticity occurred in smaller regions than what would have been anticipated from the shear strain fields $\epsilon_{x y}$. For the first angular position, the equivalent strains are lower than the yield strain, which is consistent with the linear torque vs. applied angle response. For the second angular position, some hinges were yielding, and many did not. For the third angular position on, all hinges were yielding, and the $\epsilon_{e q}$ strain increased with the applied angle. For the last angular position, the top row reached levels up to 0.5 .

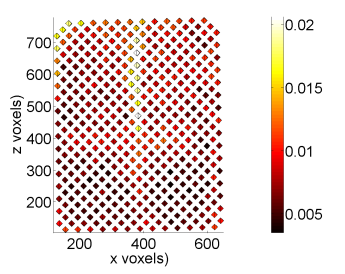

(a) $\theta=30^{\circ}$

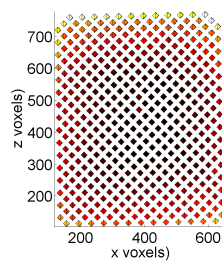

(d) $\theta=120^{\circ}$

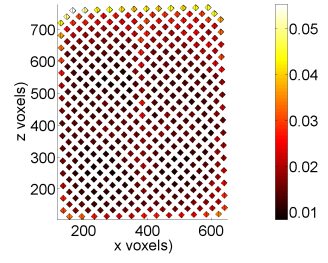

(b) $\theta=60^{\circ}$

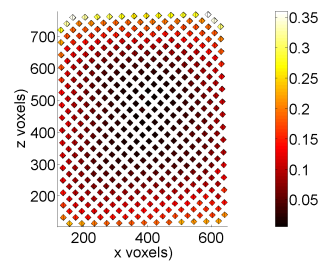

(e) $\theta=150^{\circ}$

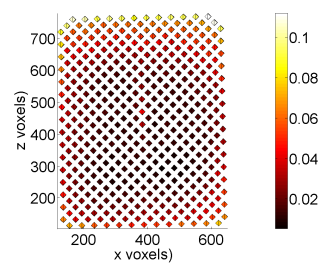

(c) $\theta=90^{\circ}$

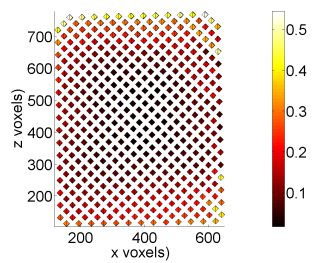

(f) $\theta=180^{\circ}$

Figure 22. Average strains $\epsilon_{e q}$ in the hinges for the different angular amplitudes. The fields are displayed on the initial configuration

If discrete models are to be considered, it is also possible to extract from the DVC measurements the displacement jumps (i.e., the displacement difference between the mean translation of the top and bottom parts of each hinge). Figure 23 shows the mean displacement jumps $\llbracket u_{y} \rrbracket$ in the out-of-plane direction for all hinges. For the last four angular positions, the distributions of $\llbracket u_{y} \rrbracket$ are identical, yet their overall amplitude increased with the applied angle. For the 
first applied angle, the distribution is totally different from the other ones. In the second angular position, a beginning of organization is observed.

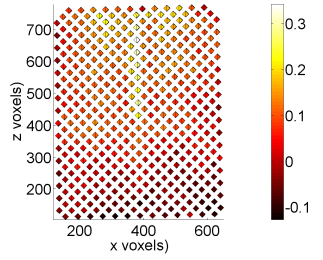

(a) $\theta=30^{\circ}$

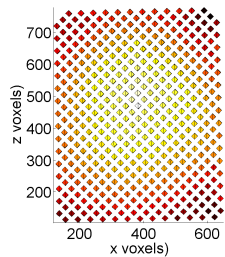

(d) $\theta=120^{\circ}$

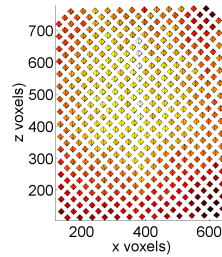

(b) $\theta=60^{\circ}$

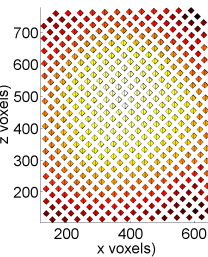

(e) $\theta=150^{\circ}$

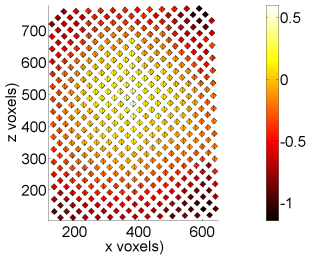

(c) $\theta=90^{\circ}$

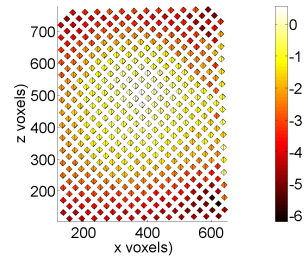

(f) $\theta=180^{\circ}$

Figure 23. Displacement jumps $\llbracket u_{y} \rrbracket$ (expressed in voxels) in the hinges for the different angular amplitudes. The fields are displayed on the initial configuration

The displacement jumps shown in Figure 23 show a shortening of the hinges. The maximum measured shortening was of the order of $300 \mu \mathrm{m}$, which is about one third of the hinge height in the reference configuration. This shortening can be understood as a Poynting effect. This interpretation is supported by the following figure, in which the relative rotation of the beams interconnected by the hinges is reported.

The rotation jumps were determined as the difference of mean rotations of the top and bottom surfaces of each hinge. Figure 24 reports the mean rotation jumps $\llbracket \omega_{y} \rrbracket$ in the out-of-plane direction for all hinges. For the last three angular positions, the distributions of $\llbracket u_{y} \rrbracket$ were identical, yet their overall amplitude increased with the applied angle. All hinges experienced large levels 
of torsional deformation. For the first three applied angles, the distributions gradually transition toward the subsequent steady state distribution.

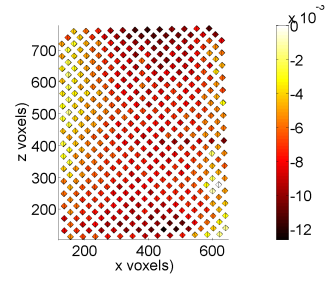

(a) $\theta=30^{\circ}$

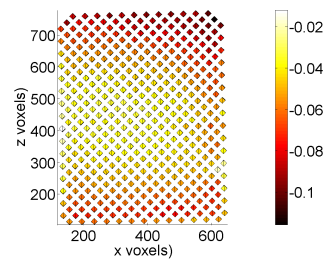

(d) $\theta=120^{\circ}$

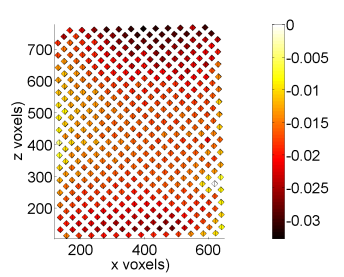

(b) $\theta=60^{\circ}$

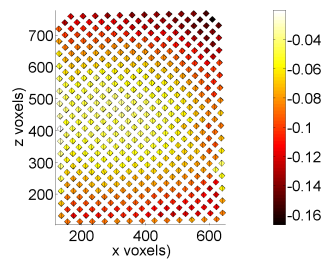

(e) $\theta=150^{\circ}$

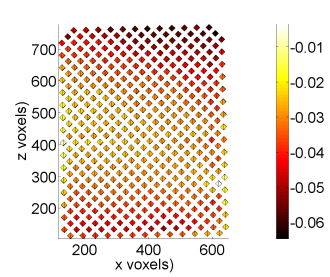

(c) $\theta=90^{\circ}$

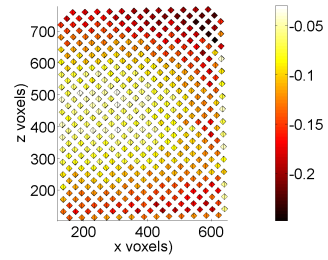

(f) $\theta=180^{\circ}$

Figure 24. Rotation jumps $\llbracket \omega_{y} \rrbracket$ in the hinges for the different angular amplitudes. The fields are displayed on the initial configuration

The distribution of the rotation jumps is similar to that of the displacement jumps shown in Figure 23. This observation shows that the displacement jumps were due to torsion of the hinge, and allows a meso-Poynting effect to be recognized in the hinges. This effect is by no means negligible because, as explained above, the hinge shortening is of the order of one third of the initial length.

\section{Analysis of the Final Scan}

The final scan corresponds to the configuration for which the applied angle was brought back to zero (Figure 2). Even though the grips recovered their reference configuration, this was not the case for the sample shape because of plasticity and possibly buckling (see Figure 2). Consequently, the final configuration 
was significantly different from the reference configuration. This difference is illustrated by the displacement field along the direction perpendicular to the pantograph plane (Figure 25(a)). Further, the maximum principal strains did not vanish (Figure 25(b)) even though the strain levels in the hinges were significantly lowered (Figure 25(c-e)) in comparison to those observed when $\theta=180^{\circ}$ (Figure $20(f)$ ). This observation explains the reason for the torque and axial loads to have very large amplitudes (Figure 3).
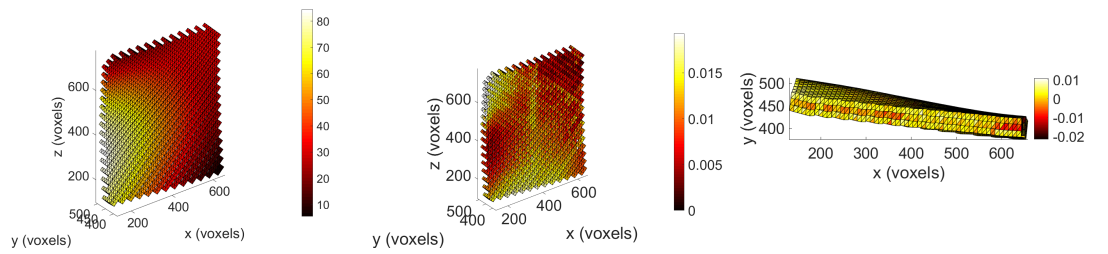

(a) $u_{y}$ (voxels)

(b) $\epsilon_{1}$

(c) $\epsilon_{x y}$

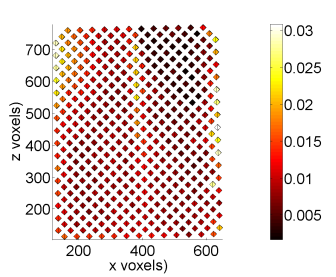

(d) $\epsilon_{e q}$

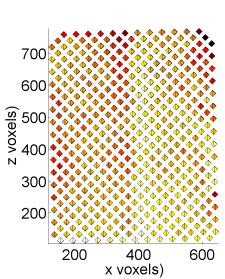

(e) $\epsilon_{x y}$

Figure 25. (a) Displacement $u_{y}$, (b) strain $\epsilon_{1}$, and (c) $\epsilon_{x y}$ fields for the final angular amplitude $(\theta=0)$ for mesoscale DVC ( $\ell_{r}=75$ voxels). The first three fields are displayed on the deformed configuration. (d) Average equivalent strain $\epsilon_{e q}$, and (e) shear strain $\epsilon_{x y}$ fields in the hinges. The last two fields are displayed on the initial configuration

In the final configuration, the displacement $\llbracket u_{y} \rrbracket$ and rotation $\llbracket \omega_{y} \rrbracket$ jump distributions (Figure 26) were different from those reached at maximum angular position (see Figures $23(\mathrm{f})$ and $24(\mathrm{f})$ ). It is worth noting that many hinges experienced positive or negative rotation jumps. 


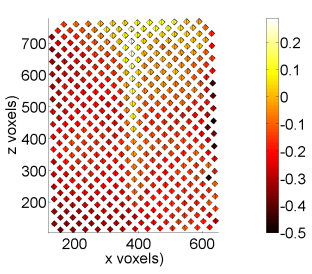

(a) $\llbracket u_{y} \rrbracket$

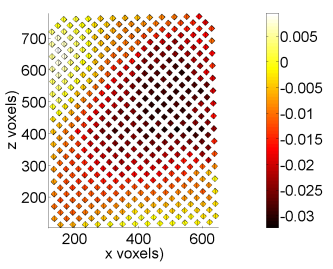

(b) $\llbracket \omega_{y} \rrbracket$

Figure 26. (a) Displacement jump $\llbracket u_{y} \rrbracket$ (expressed in voxels), and (b) rotation jump $\llbracket \omega_{y} \rrbracket$ in the hinges for the final angular amplitude $(\theta=0)$. The fields are displayed on the initial configuration

\section{Conclusion}

An in-situ torsion test on a pantographic metamaterial was analyzed via FEbased DVC (i.e., T4-DVC in the present case). Two reference scans, six scans of deformed configurations up to $180^{\circ}$ angular amplitude, and a last one when the amplitude was brought back to 0 were acquired, and the corresponding volumes were reconstructed. In the present case, no significant contrast was present in the imaged metamaterial. All these conditions made DVC analyses very challenging at the mesoscale.

A first feasibility study of DVC runs was performed for a coarse mesh at the macroscale. An initialization strategy was proposed to lower the number of iterations and achieve convergence of incremental calculations. The incremental displacement amplitudes were very large (i.e., typically \pm 30 voxels), yet displacement fields could be measured thanks to mechanical regularization. Without such strategy, DVC analyses would not have been possible since a majority of elements did not share any voxels with the pantographic structure (because of printing defects).

To perform DVC analyses at the mesoscale, the first step was to tailor the mesh to the $3 \mathrm{D}$ printed configuration. This operation was performed thanks 
to a backtracking procedure that consisted in registering the volume in the nominal configuration with that in the $3 \mathrm{D}$ printed configuration via DVC. Once the corresponding displacement field was estimated, the mesoscale mesh was backtracked. It is worth noting that the displacement field quantified the shape difference between the nominal and 3D printed geometries. At the end of this step, two meshes (i.e., one at the macroscale and another one at the mesoscale) were available.

Direct DVC runs were then successively performed on both discretizations. This was made possible by accounting for large rotations in the DVC scheme and modifying the way displacement increments were updated with the use of the nodal deformation gradient tensor. With this additional implementation, displacement amplitudes as high as \pm 180 voxels could be measured with the use of only six scans of deformed configurations. With the smallest regularization length, the displacements at the macroscopic scales led to similar registration quality than those at the mesoscale until the maximum axial load was reached. Beyond that point, the displacement fields at the mesoscopic scales were more trustworthy than their macroscale counterparts. One of the main reasons lied in the fact that the hinges experienced very high strain levels, which could only be captured by discretizations at the mesoscale.

The kinematic fields measured at the mesoscale provided a wealth of information, in particular on the shear strain, maximum principal strain and equivalent strain histories experienced by the hinges. If lumped data were preferred, displacement and rotation jumps were extracted and enabled to further interpret the macroscopic response of the tested metamaterial. From a mechanical point of view, the emergence of the Poynting effect could be analyzed herein. The Poynting effect occurred not only at macrolevel (as was already reported for such type of metamaterials [5] and corroborated herein), but also at the mesoscopic scale (i.e., at the level of the hinges), which was due 
to significant rotation jumps (i.e., meso-torsion). The Poynting effect globally involved a contraction of the hinges (and therefore of the whole metamaterial) in the direction orthogonal to the plane on which the fibers lie. Such contraction, although conceivable, would not have been quantifiable if not by means of tomographic analyses and DVC measurements.

The present data can be utilized to validate or even calibrate constitutive models of such materials at the two investigated scales. A posteriori analyses (e.g., via finite element model updating [32]) or integrated DVC [33, 34] may be selected to probe the quality of the numerical predictions against load data, displacement fields and/or 3D images. These 4D analyses will allow for very thorough comparisons at the pantographic fabric level (i.e., axial force and torque), at the macroscopic or mesoscopic levels (via displacement and strain fields), and at the microscopic level (i.e., gray level residuals).

\section{Acknowledgments}

This work has been financially supported by the French "Agence Nationale de la Recherche" through the "Investissements d'avenir" program (ANR-10-EQPX-37

MATMECA Grant). The pantographic metamaterial was kindly provided by Dr. Corinne Dupuy and Prof. Patrice Peyre (PIMM Laboratory).

\section{References}

[1] Poynting J. XXXIX. Radiation pressure. The London, Edinburgh, and Dublin Philosophical Magazine and Journal of Science 1905; 9(52): 393406.

[2] Poynting J. On pressure perpendicular to the shear planes in finite pure shears, and on the lengthening of loaded wires when twisted. Proceedings of the Royal Society of London 1909; A82(557): 546-559. 
[3] Poynting J. The changes in length and volume of an Indian-rubber cord when twisted. India-Rubber Journal 1913; October 4: 6.

[4] Georgievskii D. Types of Physical Nonlinearity in the Theory of Constitutive Relations and the Generalized Poynting Effect. Springer International Publishing, 2019. pp. 129-136.

[5] Misra A, Lekszycki T, Giorgio I et al. Pantographic metamaterials show atypical Poynting effect reversal. Mechanics Research Communications 2018; 89: 6-10.

[6] Barchiesi E, Spagnuolo M and Placidi L. Mechanical metamaterials: a state of the art. Mathematics and Mechanics of Solids 2019; 24(1): 212-234.

[7] dell'Isola F, Seppecher P, Alibert JJ et al. Pantographic metamaterials: an example of mathematically driven design and of its technological challenges. Continuum Mechanics and Thermodynamics 2019; 31(4): 851-884.

[8] Turco E, dell'Isola F, Cazzani A et al. Hencky-type discrete model for pantographic structures: numerical comparison with second gradient continuum models. Zeitschrift für angewandte Mathematik und Physik 2016; 67: 85.

[9] Barchiesi E and Placidi L. A Review on Models for the 3D Statics and 2D Dynamics of Pantographic Fabrics. Springer Singapore, 2017. pp. 239-258.

[10] Giorgio I, Rizzi N and Turco E. Continuum modelling of pantographic sheets for out-of-plane bifurcation and vibrational analysis. Proceedings of the Royal Society A 2017; 473: 20170636.

[11] Turco E. How the Properties of Pantographic Elementary Lattices Determine the Properties of Pantographic Metamaterials. Cham: Springer, 2019. pp. 489-506. 
[12] Giorgio I, Rizzi N, Andreaus U et al. A two-dimensional continuum model of pantographic sheets moving in a 3D space and accounting for the offset and relative rotations of the fibers. Mathematics and Mechanics of Complex Systems 2019; 7(4): 311-325.

[13] dell'Isola F, Cuomo M, Greco L et al. Bias extension test for pantographic sheets: numerical simulations based on second gradient shear energies. Journal of Engineering Mathematics 2017; 103(1): 127-157.

[14] Turco E, Misra A, Pawlikowski M et al. Enhanced piola-hencky discrete models for pantographic sheets with pivots without deformation energy: Numerics and experiments. International Journal of Solids and Structures 2018; 147: 94-109.

[15] Hild F, Misra A and dell'Isola F. Multiscale DIC applied to Pantographic Structures. Experimental Mechanics 2020; (DOI: 10.1007/s11340-02000636-y).

[16] Ganzosch G, Hoschke K, Lekszycki T et al. 3D-Measurements of 3DDeformations of Pantographic Structures. Technische Mechanik 2018; 38(3): 233-245.

[17] Barchiesi E, Ganzosch G, Liebold C et al. Out-of-plane buckling of pantographic fabrics in displacement-controlled shear tests: experimental results and model validation. Continuum Mechanics and Thermodynamics 2018; 31: 33-45.

[18] Dufour JE, Hild F and Roux S. Shape, Displacement and Mechanical Properties from Isogeometric Multiview Stereocorrelation. Journal of Strain Analysis for Engineering Design 2015; 50(7): 470-487. 
[19] dell'Isola F, Lekszycki T, Spagnuolo M et al. Experimental methods in pantographic structures. In dell'Isola F and Steigmann D (eds.) Discrete and Continuum Models for Complex Metamaterials. Cambridge University Press, pp. 263-297.

[20] Maire E and Withers PJ. Quantitative X-ray tomography. International Materials Reviews 2014; 59(1): 1-43.

[21] Taillandier-Thomas T, Roux S, Morgeneyer $\mathrm{T}$ et al. Localized strain field measurement on laminography data with mechanical regularization. Nuclear Instruments and Methods in Physics Research Section B 2014; 324: $70-79$.

[22] dell'Isola F, Seppecher P, Spagnuolo M et al. Advances in Pantographic Structures: Design, Manufacturing, Models, Experiments and Image Analyses. Continuum Mechanics and Thermodynamics 2019; 31(4): 12311282.

[23] Leclerc H, Neggers J, Mathieu F et al. Correli 3.0. IDDN.FR.001.520008.000.S.P.2015.000.31500, Agence pour la Protection des Programmes, Paris (France), 2015.

[24] Roux S and Hild F. Stress intensity factor measurements from digital image correlation: post-processing and integrated approaches. International Journal of Fracture 2006; 140(1-4): 141-157.

[25] Guery A, Latourte F, Hild F et al. Characterization of SEM speckle pattern marking and imaging distortion by Digital Image Correlation. Measurement Science and Technology 2014; 25(1): 015401. 
[26] Neggers J, Blaysat B, Hoefnagels JPM et al. On image gradients in digital image correlation. International Journal for Numerical Methods in Engineering 2016; 105(4): 243-260.

[27] Passieux JC and Bouclier R. Classic and inverse compositional GaussNewton in global DIC. International Journal for Numerical Methods in Engineering 2019; 119(6): 453-468.

[28] Mendoza A, Schneider J, Parra E et al. The correlation framework: Bridging the gap between modeling and analysis for 3D woven composites. Composite Structures 2019; 229: 111468.

[29] Buljac A, Jailin C, Mendoza A et al. Digital Volume Correlation: Review of Progress and Challenges. Experimental Mechanics 2018; 58(5): 661-708.

[30] Buljac A, Taillandier-Thomas T, Helfen L et al. Evaluation of measurement uncertainties of digital volume correlation applied to laminography data. Journal of Strain Analysis for Engineering Design 2018; 53: 49-65.

[31] Spagnuolo M, Barcz K, Pfaff A et al. Qualitative pivot damage analysis in aluminum printed pantographic sheets: Numerics and experiments. Mechanics Research Communications 2017; 83: 47-52.

[32] Grédiac M and Hild F (eds.) Full-Field Measurements and Identification in Solid Mechanics. London (UK): ISTE / Wiley, 2012.

[33] Hild F, Bouterf A, Chamoin L et al. Toward 4D Mechanical Correlation. Advanced Modeling and Simulation in Engineering Sciences 2016; 3(1): 126.

[34] Buljac A, Trejo-Navas VM, Shakoor M et al. On the calibration of elastoplastic parameters at the microscale via X-ray microtomography and 
digital volume correlation for the simulation of ductile damage. European Journal of Mechanics - A/Solids 2018; 72: 287-297. 